\title{
ESTIMACION DE PARÁMETROS HIDRODINÁMICOS EN UN ACUÍFERO VOLCÁNICO LIBRE FRACTURADO EN COSTA RICA. PARTE II. MODELO DE DOBLE POROSIDAD
}

\author{
ESTIMATION OF HYDRODINAMICS PARAMETERS IN A VOLCANIC \\ FRACTURED PHREATIC AQUIFER IN COSTA RICA. PART II. \\ DOUBLE POROSITY MODEL
}

\author{
Julio Macías ${ }^{1}$ y Asdrubal Vargas ${ }^{2}$ \\ ${ }^{1}$ Consultor en Geomecánica \\ ${ }^{2}$ Centro de Servicio Exploración Subterránea, Instituto Costarricense de \\ Electricidad (I.C.E.), Apdo. 10032-1000, San José, Costa Rica \\ *Autor para contacto: jmaciasa@netscape.net
}

(Recibido: 04/08/2016; aceptado: 17/04/2017)

\begin{abstract}
MIM 1D transport model was successfully applied to simulate the asymmetric behavior observed in three breakthrough curves of tracer tests performed under natural gradient conditions in a phreatic fractured volcanic aquifer. The transport parameters obtained after adjustment with a computer program, suggest that only $50 \%$ of the total porosity effectively contributed to the advective-dispersive transport (mobile fraction) and the other $50 \%$ behaved as a temporary reservoir for the tracer (immobile fraction). The estimated values of hydraulic properties and MIM model parameters are within the range of values reported by other researchers. It was possible to establish a conceptual and numerical framework to explain the three-tracer tests curves behavior, despite the limitations in quality and quantity of available field information.

Keywords: Fractured aquifer, analytic model MIM, tracer test, spring, hydrogeology.

RESUMEN: En este trabajo se aplicó el modelo de transporte MIM 1D para simular de manera satisfactoria el comportamiento asimétrico observado en las curvas de llegada de tres ensayos de trazador en condición de gradiente natural realizados en un acuífero libre desarrollado en lavas fracturadas. Los parámetros de transporte obtenidos a partir del empleo de un programa de computador (que permitió ajustar los datos de campo con una curva teórica), sugieren que solamente el $50 \%$ de la porosidad total contribuyó efectivamente al transporte advectivo-dispersivo (fracción móvil), y que el otro $50 \%$ se comportó como un reservorio transitorio para el trazador (fracción inmóvil), siendo liberado o transferido hacia la fracción móvil de manera gradual. Los valores de los parámetros representativos del modelo MIM obtenidos en este trabajo, así como las propiedades hidráulicas estimadas para el acuífero se encuentran dentro del rango de valores reportados por otros autores. Esto es, que a pesar de las limitaciones en la cantidad y calidad de información de campo disponible fue posible establecer un marco conceptual y numérico para explicar la asimetría observada en los tres ensayos.

Palabras clave: Acuífero fracturado, modelo analítico MIM, ensayo trazador, manantial, hidrogeología.
\end{abstract}




\section{INTRODUCCIÓN}

Este trabajo es la continuación de los análisis iniciados por Macías, Vargas y Frutos (2016). Dicha publicación incluye la interpretación de tres ensayos de trazador a través de modelos unidimensionales de porosidad simple. En este trabajo se presenta el análisis empleando un modelo de doble porosidad.

Las curvas de concentración obtenidas en los ensayos referidos mostraron un comportamiento claramente asimétrico, caracterizado por un arribo bastante pronunciado seguido de un decaimiento gradual pos-pico. Ese comportamiento ha sido documentado por varios autores tanto para suelos no saturados, suelos agregados y suelos fracturados o con macroporos (van Genuchten, 1981), así como para medios rocosos fracturados (Raven, Novakowski y Lapcevic, 1988; Fetter,1993). Diferentes modelos conceptuales y matemáticos han sido propuestos para simular ese comportamiento. Estos modelos pueden agruparse en dos categorías dependiendo de las consideraciones para explicar los procesos que generan la asimetría observada (van Genuchten, 1981). Para la primera categoría, esta asimetría es producto de un proceso de transporte bajo condiciones de desequilibrio físico. Estos es, se basa en consideraciones puramente físicas en las que se asume la existencia de dos zonas de fluido, una móvil que asume el transporte advectivo-dispersivo y otra inmóvil hacia la cual migra el soluto por difusión. Esta zona inmóvil retiene temporalmente al soluto para liberarlo posteriormente. En esta categoría se encuentra el denominado modelo MIM (Móvil-Inmóvil). La segunda categoría, por otro lado se basa en consideraciones químicas, y asume que el transporte ocurre en condiciones de desequilibrio químico, en el cual se presentan dos tipos de sitios de adsorción, los cuales retienen y liberan el soluto a diferentes tasas, una de manera instantánea y otra dependiente del tiempo, es decir, es un proceso no equilibrado de adsorción.

En este trabajo se presentan los resultados de las simulaciones para los tres ensayos de trazador a través del modelo MIM unidimensional. Este modelo fue escogido por las siguientes razones: la na- turaleza no reactiva del trazador empleado (cloruro de sodio), y por contar con información de apenas un punto de inyección y solamente un punto de observación. Es decir en este trabajo se restringe el análisis a una idealización unidimensional.

\section{FORMULACIÓN CONCEPTUAL, MATEMÁTICA Y SOLUCIÓN ANALÍTICA DEL MODELO MIM}

Desde el punto de vista conceptual, el modelo MIM define la existencia de dos zonas de fluido: una móvil en la cual ocurre el transporte advectivo-dispersivo, y otra inmóvil (o con cierta movilidad) que no contribuye al transporte del soluto sino que lo retiene temporalmente para después liberarlo. En el caso de fracturas, este concepto se aplica a los efectos provocados por la rugosidad de las mismas. Esto es, la rugosidad genera dos fenómenos: zonas de movimiento restringido cuando las paredes rugosas entran en contacto, y zonas de vórtices a lo largo del eje de las fracturas que mantienen retenido al soluto. Este soluto será inmovilizado mientras exista una mayor concentración en la fracción móvil, y será liberado una vez esa concentración sea menor a la de la fracción inmóvil; es decir, el soluto será transferido (liberado) desde la fracción inmóvil hacia la fracción móvil (Raven, Novakowski y Lapcevic, 1988). La tasa de transferencia entre las dos zonas de movilidad es conceptualizada como un proceso de primer orden y dependiente de la diferencia de concentración entre ambas zonas.

La formulación matemática empleada en este trabajo está basada en la formulación presentada por van Genuchten (1981), la cual emplea las ecuaciones de balance de masa del soluto para las fracciones móvil e inmóvil, y son acopladas a través de un factor de transferencia. Ese modelo se muestra a continuación, manteniendo la misma simbología empleada por van Genuchten (1981).

$$
\begin{aligned}
& \theta_{m} \frac{\partial c_{m}}{\partial t}+\theta_{i m} \frac{\partial c_{i m}}{\partial t}=\theta_{m} D \frac{\partial^{2} c_{m}}{\partial x^{2}}-\theta_{m} v_{m} \frac{\partial c_{m}}{\partial x}(\text { Ec. } 1) \\
& \theta_{i m} \frac{\partial c_{i m}}{\partial t}=\alpha\left(c_{m}-c_{i m}\right)
\end{aligned}
$$




$$
\begin{aligned}
& v_{m}=\frac{q}{\theta_{m}}=\frac{v}{\phi_{m}} \\
& \phi_{m}=\frac{\theta_{m}}{\theta} \quad\left(\theta=\theta_{m}+\theta_{i m}\right)
\end{aligned}
$$

Donde:

$\theta=$ contenido volumétrico de agua $\left(\mathrm{L}^{3} / \mathrm{L}^{3}\right)$

$\theta_{\mathrm{m}}=$ contenido volumétrico de agua correspondiente a la fracción móvil $\left(\mathrm{L}^{3} / \mathrm{L}^{3}\right)$

$\theta_{\text {im }}=$ contenido volumétrico de agua correspondiente a la fracción inmóvil $\left(\mathrm{L}^{3} / \mathrm{L}^{3}\right)$

$\phi_{\mathrm{m}}=$ fracción de agua móvil (adimensional)

$\mathrm{c}_{\mathrm{im}}=$ concentración de soluto en fracción inmóvil $\left(\mathrm{M} / \mathrm{L}^{3}\right)$

$\mathrm{c}_{\mathrm{m}}=$ concentración de soluto en fracción mó$\operatorname{vil}\left(\mathrm{M} / \mathrm{L}^{3}\right)$

$v_{\mathrm{m}}=$ velocidad de poros de la fracción móvil $(\mathrm{L} / \mathrm{T})$

$v=$ velocidad de poros correspondiente a toda la fase líquida $(\mathrm{L} / \mathrm{T})$

$q=\mathrm{vd}=$ flujo volumétrico de fluido o velocidad de Darcy $\left(\mathrm{L}^{3} / \mathrm{L}^{2} \mathrm{~T}\right)$

$D=$ dispersión $\left(\mathrm{L}^{2} / \mathrm{T}\right)$

$\alpha=$ constante de transferencia de soluto entre las fracciones móvil e inmóvil (1/T)

$x, t=$ coordenadas de distancia (L) y tiempo

(T) respectivamente

El parámetro $\alpha$ en la ecuación 2 corresponde con la constante de transferencia y representa la rapidez con que ocurre el intercambio de masa. El factor $\phi_{\mathrm{m}}$ en la ecuación 4 representa la fracción del volumen de fluido que efectivamente trasporta el soluto en relación al volumen total del fluido.

Para simplificar la solución matemática de estas ecuaciones, se introducen las siguientes variables adimensionales.

$$
\begin{aligned}
& T=\frac{v t}{L}=\frac{v_{m} t \phi_{m}}{L} \\
& Z=\frac{x}{L} \\
& P=\frac{v_{m} L}{D}
\end{aligned}
$$

$$
\begin{aligned}
& \omega=\frac{\alpha L}{q}=\frac{\alpha L}{\theta_{m} v_{m}} \\
& \beta=\frac{\phi_{m} R_{m}}{R} \\
& c_{1}=\frac{c_{m}-c_{i}}{c_{0}-c_{i}} \\
& c_{2}=\frac{c_{\mathrm{i} m}-c_{i}}{c_{0}-c_{i}}
\end{aligned}
$$

Donde:

$\mathrm{c}_{\mathrm{i}}=$ concentración de fondo $\left(\mathrm{M} / \mathrm{L}^{3}\right)$

$\mathrm{c}_{0}=$ concentración de inyección $\left(\mathrm{M} / \mathrm{L}^{3}\right)$

$\mathrm{c}_{1} \mathrm{c}_{2}=$ concentraciones normalizadas en fracciones móvil e inmóvil respectivamente $\left(\mathrm{M} / \mathrm{L}^{3}\right)$

$\mathrm{T}, \mathrm{Z}=$ coordenadas de tiempo y espacio normalizadas

$\mathrm{L}=$ distancia al punto de observación (L)

$\mathrm{P}=$ número de Peclet en relación a velocidad de fracción móvil

$\mathrm{R}, \mathrm{R}_{\mathrm{m}}=$ factores de retardo en relación a todo el fluido y fracción móvil respectivamente

$\beta=$ fracción móvil de fluido, en este caso es idéntico a $\phi_{\mathrm{m}}$ pues $\mathrm{R}=\mathrm{R}_{\mathrm{m}}=1$

$\omega=$ constante de transferencia normalizada a tiempo medio de flujo según $v_{\mathrm{d}}$

El parámetro @ es también conocido como número I de Damkohler, y representa la relación entre la escala de tiempo en que ocurre el flujo del fluido (macroscópica) y la escala de tiempo en que ocurre la transferencia de masa. El parámetro $\mathrm{T}$ al ser normalizado en relación al tiempo medio de flujo del fluido según la velocidad de poros v, representa el número de volúmenes de poro desplazados.

Para el interés de este trabajo, las ecuaciones 1 y 2 deben resolverse para una condición de contorno tipo Dirichlet, es decir con concentración impuesta. La solución con esta condición asegura que el resultado sea mostrado en términos de concentración en efluente y no en términos de 
concentración residual. Dicha solución analítica puede ser revisada en van Genuchten (1981). Este mismo autor adjunta el código de programación en lenguaje FORTRAN de un programa denominado CFITIM. Este programa permite la solución de las ecuaciones 1 y 2 a través de un proceso de integración numérica. Los datos de entrada son: (a) la curva de concentración de campo normalizada en relación a la concentración de inyección (este procedimiento es diferente al seguido por Macías, Vargas y Frutos (2016) donde se normalizó en relación a la concentración máxima medida), (b) el tiempo de campo normalizado a tiempo medio de flujo según la velocidad de poros v, es decir se ingresan números de volumen de poro desplazados y (c) valores iniciales para los parámetros $\mathrm{P}, \mathrm{R}, \beta$, $\mathrm{y}$ tiempo de duración del pulso de inyección normalizado según v (T1). El programa cuenta adicionalmente con una rutina de optimización de los parámetros $\mathrm{P}, \mathrm{R}, \beta$, $⿴ \mathrm{y}$ T1 de manera simultánea, a través de un ajuste por mínimos cuadrados entre la curva de campo y la curva teórica. El usuario también puede definir valores fijos para estos parámetros. El programa CFITIM fue parcialmente modificado por los autores de esta publicación para agilizar el ingreso de los datos. Más adelante se hace referencia a este programa como CFITIM-Modificado.

\section{CONCENTRACIÓN DE INYECCIÓN Y CONCENTRACIÓN DE SALIDA}

Debido a que la solución de las ecuaciones 1 y 2 se encuentra normalizada en relación a la concentración de inyección, este valor deber ser definido de manera explícita. Para el cálculo de la concentración de trazador que efectivamente es transmitida desde la perforación de inyección hacia el acuífero, se empleó el modelo propuesto por Bouyère, Carabin y Dassargues (2005), resumido en las ecuaciones 12 y 13.

$$
\begin{aligned}
& C_{w}=\frac{Q_{\text {in }} C_{\text {in }}-\left(Q_{\text {in }} c_{\text {in }}-Q_{\text {out }} C_{o}\right) \exp \left(-\frac{Q_{\text {out }}}{V_{w}}(t)\right)}{Q_{\text {out }}} \\
& Q_{\text {out }}=Q_{\text {in }}+Q_{t}^{\text {in }}
\end{aligned}
$$

Donde:

$\mathrm{V}_{\mathrm{w}}=$ volumen de agua presente en el pozo al momento de la inyección $\left(\mathrm{L}^{3}\right)$

$\mathrm{t}=$ tiempo medido a partir del inicio de la inyección $(\mathrm{T})$

$\mathrm{C}_{\mathrm{w}}=$ concentración de salida del pozo de inyección hacia el acuífero $\left(\mathrm{M} / \mathrm{L}^{3}\right)$

$\mathrm{C}_{\text {in }}=$ concentración inyectada al pozo $(\mathrm{M} / \mathrm{L} 3)$

$\mathrm{C}_{0}=$ concentración de fondo en pozo de inyección $\left(\mathrm{M} / \mathrm{L}^{3}\right)$

$\mathrm{Q}_{\text {in }}=$ flujo en que solución es inyectada al pozo de inyección $\left(\mathrm{L}^{3} / \mathrm{T}\right)$

$\mathrm{Q}_{\text {out }}=$ flujo en que el fluido sale del pozo de inyección hacia el acuífero $\left(\mathrm{L}^{3} / \mathrm{T}\right)$

$\mathrm{Q}_{\mathrm{t}}^{\mathrm{in}}=$ flujo en que el fluido entra al pozo de inyección desde el acuífero $\left(\mathrm{L}^{3} / \mathrm{T}\right)$. Este flujo es variable y depende tanto de $\mathrm{Q}_{\mathrm{in}}$ como de la geometría del pozo, longitud de rejilla y de la velocidad de Darcy en el acuífero. Más detalles sobre este factor pueden ser revisados en la publicación referida.

Debido a las condiciones variables de flujo para los tres ensayos analizados en este trabajo, como se indicó en Macías, Vargas y Frutos (2016), el valor $\mathrm{Q}_{\mathrm{t}}^{\text {in }}$ fue actualizado a través de un proceso iterativo. En el cuadro 1 se presentan los valores de concentración máxima de salida obtenidos y corregidos por la concentración de fondo. Estas son las concentraciones estimadas, que fueron efectivamente inyectadas, para cada ensayo, $\mathrm{y}$ corresponden con el valor $\mathrm{C}_{\mathrm{o}}$ en la ecuación 12 .

\section{PARÁMETROS Y SIMULACIÓN DE CURVAS DE LLEGADA CON MODELO MIM}

Inicialmente se procedió al ajuste de los datos del Ensayo 1. Para este efecto se utilizaron los valores $\mathrm{q}=69,03 \mathrm{~m} / \mathrm{d}$ para la velocidad de Darcy y $\mathrm{H}=16,7 \mathrm{~m}$ para la columna de agua en el pozo de inyección, de acuerdo con los resultados mostrados en el cuadro 5 de Macías, Vargas y Frutos (2016) y reproducido como cuadro 6 en este trabajo. El valor de q escogido corresponde con el valor máximo de permeabilidad $(\mathrm{k}=2092 \mathrm{~m} / \mathrm{d})$ estimado para el acuífero. El análisis pudo realizarse sin embargo con cualquier valor dentro del rango mostrado en el cuadro referido. Para estos 
Cuadro 1.

Concentración de salida (inyectada)

\begin{tabular}{cccccc}
\hline Ensayo & $\begin{array}{c}\text { Masa inyectada } \\
(\mathrm{Kg})\end{array}$ & $\begin{array}{c}\text { Volumen inyectado } \\
(\mathrm{l})\end{array}$ & $\begin{array}{c}\text { Tiempo inyección } \\
(\mathrm{min})\end{array}$ & $\begin{array}{c}\text { Concentración de } \\
\text { inyección Cin }(\mathrm{mg} / \mathrm{l})\end{array}$ & $\begin{array}{c}\text { Concentración de salida } \\
\mathrm{Cw}(\mathrm{mg} / \mathrm{l})\end{array}$ \\
\hline 1 & 45,5 & 185 & 20 & 245946 & 37218 \\
2 & 90,1 & 185 & 20 & 487027 & 151051 \\
3 & 90,1 & 185 & 20 & 487027 & 148198 \\
\hline
\end{tabular}

valores de q y $\mathrm{H}$ se procedió a calcular la concentración de salida del trazador empleando las ecuaciones 12 y 13. Este es el valor mostrado en el cuadro 1, y fue empleado para normalizar los datos de concentración de campo para la entrada al programa CFITIM-Modificado. Seguidamente, se realizaron diferentes ajustes de la curva de llegada para diferentes valores de velocidad de poros (v), definiendo los valores de T para cada caso. Se escogió como el ajuste más satisfactorio aquel que produjera un factor de retardo unitario $(\mathrm{R}=1)$ debido al carácter no reactivo del trazador utilizado. Con la velocidad de poros correspondiente a $\mathrm{R}=1$ se estimó el valor de la porosidad para la fase fluida.

Para los Ensayos 2 y 3 se empleó un proceso iterativo de solución. En este caso, con el valor de porosidad obtenida en el paso anterior, se procedió a calcular la velocidad de Darcy (vd) y la altura $\mathrm{H}$ para diferentes valores de velocidad de poros (v). Con cada nuevo par de valores (vd y H) la concentración de salida fue actualizada; seguidamente se procedió al ajuste con el programa CFITIM-Modificado. Este proceso iterativo se repitió hasta obtener el valor de la velocidad de poros que arrojara un valor unitario para el factor de retardo. Los ajustes finales obtenidos son mostrados gráficamente en la figura 1.
Las curvas obtenidas muestran un ajuste muy satisfactorio tanto para la llegada del trazador como para la cola de decaimiento. Este comportamiento no fue posible reproducirlo con los modelos advectivos-dispersivos de porosidad simple mostrados en Macías, Vargas y Frutos (2016). Para comparación se muestra en la figura 1 , el ajuste obtenido con el modelo de Kreft y Zuber (1978). En el cuadro 2 se presenta el resumen de los parámetros obtenidos junto a la velocidad de poros requerida para obtener un factor de retardo unitario $(\mathrm{R}=1)$. Se incluyen en el mismo cuadro los valores de P y v para los modelos de Kreft y Zuber (1978) y Lapidus y Amundson (1952) obtenidos por Macías, Vargas y Frutos (2016).

En el cuadro 3 se resumen los valores de dispersión obtenidos a partir de la ecuación 7 , y los valores correspondientes de dispersividad calculados en relación a la velocidad poros (v). También se incluyen las dispersividades para los modelos de Kreft y Zuber (1978) y Lapidus y Amundson (1952).

En el cuadro 4 se muestran los valores de velocidad de Darcy, velocidad de poros, velocidad de poros de la fase líquida, fracción de fluido móvil $\left(\phi_{\mathrm{m}}\right)$ y la constante de transferencia $(\alpha)$. El parámetro $\alpha$ fue calculado con la ecuación 8 y los

Cuadro 2

Parámetros optimizados para curvas ajustadas con $\mathrm{R}=1$

\begin{tabular}{cccccccccc}
\hline Ensayo & \multicolumn{3}{c}{ MIM R=1 } & \multicolumn{3}{c}{ Kreft Zuber 1978 } & \multicolumn{2}{c}{ Lapidus y Amundson 1952 } \\
\hline & $\mathrm{P}$ & $\mathrm{R}$ & $\beta$ & $\omega$ & $\mathrm{v}(\mathrm{m} / \mathrm{d})$ & $\mathrm{P}$ & $\mathrm{v}(\mathrm{m} / \mathrm{d})$ & $\mathrm{P}$ & $\mathrm{v}(\mathrm{m} / \mathrm{d})$ \\
1 & 29,2 & 1 & 0,491 & 1,567 & 848,9 & 21 & 1622 & 14 & 1842 \\
2 & 251,7 & 1 & 0,517 & 2,046 & 410,5 & 235 & 783 & 216 & 840 \\
3 & 91,4 & 1 & 0,542 & 1,700 & 420,2 & 85 & 755 & 74 & 807 \\
\hline
\end{tabular}



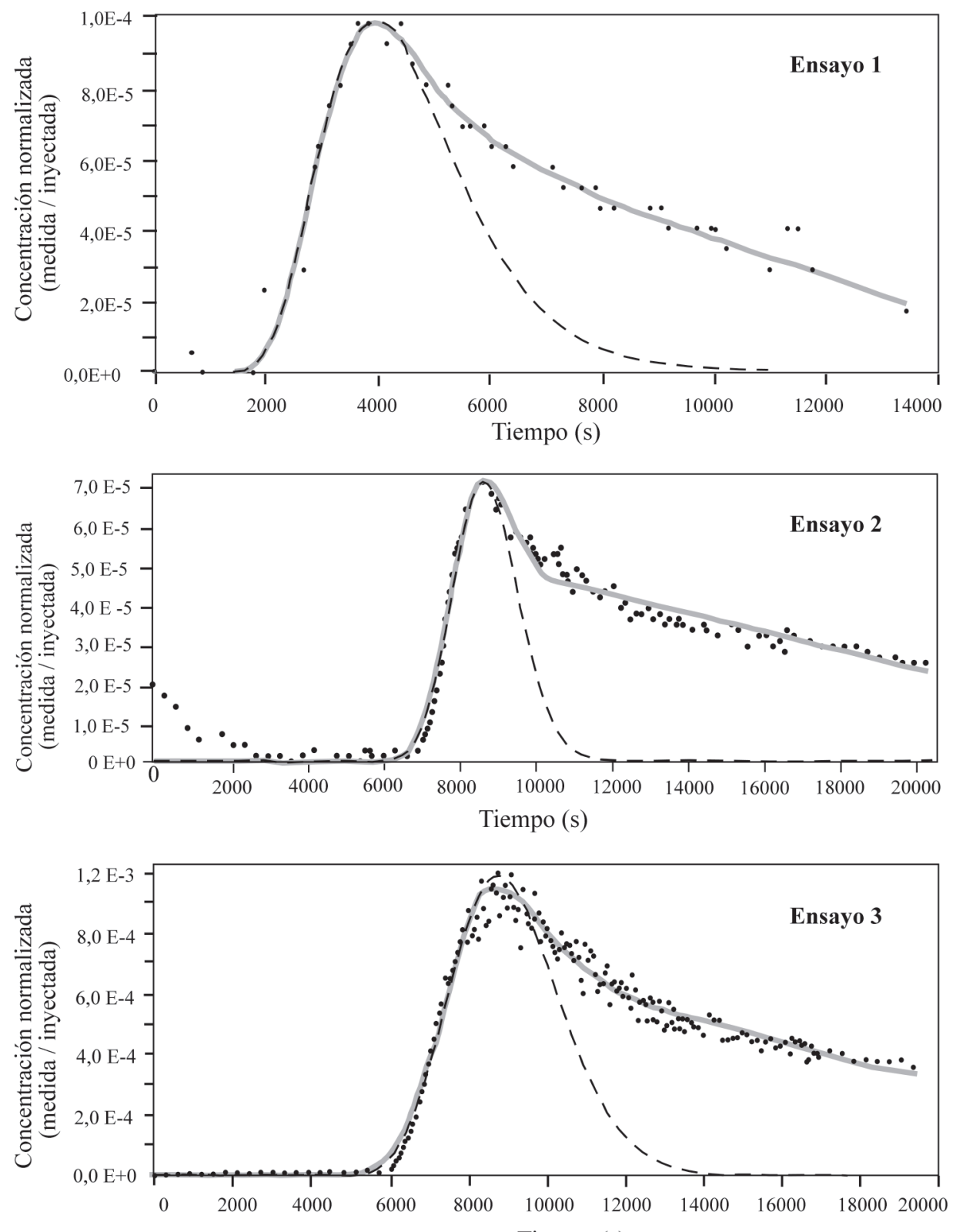

- - Datos de campo

Tiempo (s)

Ajuste programa CFITIM-Modificado

- - - Ajuste modelo Kreft \& Zuber 1978

Fig. 1: Ajuste de curva de llegada para los ensayos 1, 2 y 3 .

valores $₫$ del cuadro 2. Esta ecuación relaciona $₫$ y $\alpha$ con la velocidad de Darcy $\left(\mathrm{v}_{\mathrm{d}}\right)$.

Para el Ensayo 1 los resultados obtenidos indican que el trasporte ocurrió bajo condiciones de mayor velocidad de poros y mayor dispersión.
Esta misma observación fue realizada por Macías, Vargas y Frutos (2016) a partir de modelos de porosidad simple. La mayor velocidad, producto de un mayor caudal, permite el acceso del soluto a más caminos de flujo, tornando el transporte más 
Cuadro 3

Valores de dispersividad longitudinal.

\begin{tabular}{cccccccc}
\hline Ensayo & $\begin{array}{c}v d \\
(\mathrm{~m} / \mathrm{d})\end{array}$ & $\begin{array}{c}v \\
(\mathrm{~m} / \mathrm{d})\end{array}$ & $\begin{array}{c}v m \\
(\mathrm{~m} / \mathrm{d})\end{array}$ & $\begin{array}{c}\text { Dispersión } \\
\left(\mathrm{m}^{2} / \mathrm{d}\right)\end{array}$ & $\begin{array}{c}\text { MIM Dispersividad } \\
(\mathrm{m})\end{array}$ & $\begin{array}{c}\text { Kreft y Zuber }(1978) \\
\text { Dispersividad }(\mathrm{m})\end{array}$ & $\begin{array}{c}\text { LapidusyAmundson(1952) } \\
\text { Dispersividad }(\mathrm{m})\end{array}$ \\
\hline 1 & 69,03 & 848,9 & 1728,9 & 4665,7 & 5,50 & 3,75 & 5,63 \\
2 & 33,45 & 410,5 & 794,0 & 248,6 & 0,61 & 0,34 & 0,36 \\
3 & 34,16 & 420,2 & 775,3 & 668,4 & 1,59 & 0,93 & 1,06 \\
\hline
\end{tabular}

tortuoso. Este comportamiento es reflejado en los valores de dispersividad y número de Peclet. También para este ensayo se obtuvo el menor valor de la fracción móvil. La disminución de la fracción móvil con el aumento de la velocidad ha sido documentada por ensayos de campo y laboratorio (Raven, Novakowski y Lapcevic, 1988; Griffioen, Barry y Parlange, 1998; Mayes et al., 2003). Esto se interpreta como producto de la mayor turbulencia en las paredes de las fracturas. El parámetro $₫$ calculado para el Ensayo 1 presenta el menor valor, indicando que para este ensayo la tasa de transferencia ocurrió más aceleradamente en relación a la velocidad del fluido. El valor de $\alpha$ calculado es el mayor, confirmando este comportamiento, es decir, mayor rapidez en la mezcla y transferencia entre las fracciones móvil e inmóvil. El aumento del valor de la constante de transferencia con la velocidad ha sido documentado por Raven, Novakowski y Lapcevic (1988) y Griffioen, Barry y Parlange (1998). Este parámetro también pues ser afectado por la geometría del medio (Gvirtzman et al. [1988]).

Para el Ensayo 2 los resultados obtenidos indican que el trasporte ocurrió bajo condiciones de menor velocidad de poros y por lo tanto se obtuvo una dispersión menor. Este mismo comportamiento es indicado por el análisis con modelos de porosidad simple (Macías, Vargas y Frutos,
2016). Esto refleja el hecho de que para menor caudal, el movimiento del fluido es concentrado en caminos preferenciales disminuyendo la probabilidad de que el soluto encuentre fracturas cerradas o discontinuas. Esta menor velocidad también se ve reflejada en un mayor valor de fracción móvil y un menor valor de la constante de transferencia. El mayor valor de $₫$ en relación al Ensayo 1 indica que la trasferencia ocurrió a una escala de tiempo mayor en comparación al flujo, interpretado esto como una menor turbulencia durante el transporte.

Para el caso del Ensayo 3, la velocidad de poros de toda la fase líquida es ligeramente mayor a la del Ensayo 2, y esto explicaría la mayor dispersión observada en dicho ensayo. Sin embargo para este caso se estimó una mayor fracción de fluido móvil y un menor valor de $\alpha$. Es decir, para este ensayo por contar con una velocidad de poros ligeramente mayor debería esperarse una menor fracción móvil y una mayor tasa de transferencia. Se plantea como hipótesis de trabajo que la mayor concentración de trazador posiblemente inyectada en este ensayo (ver Macías, Vargas y Frutos [2016]) produjo un menor gradiente de concentración entre las fracciones móvil e inmóvil, generando un perfil de concentración más homogéneo dentro de la fractura; facilitando así que una fracción mayor participara en el traspor-

\section{Cuadro 4}

Fracción de fluido móvil y tasas de transferencia.

\begin{tabular}{cccccc}
\hline Ensayo & $v_{d}(\mathrm{~m} / \mathrm{d})$ & $v(\mathrm{~m} / \mathrm{d})$ & $v_{m}(\mathrm{~m} / \mathrm{d})$ & $\phi_{\mathrm{m}}$ & $\alpha(1 / \mathrm{d})$ \\
\hline 1 & 69,03 & 848,9 & 1728,9 & 0,491 & 1,37 \\
2 & 33,45 & 410,5 & 794,0 & 0,517 & 0,87 \\
3 & 34,16 & 420,2 & 775,3 & 0,542 & 0,74 \\
\hline
\end{tabular}


Cuadro 5

Parámetros hidráulicos estimados según resultados de MIM.

\begin{tabular}{ccccccccc}
\hline Ensayo & $\mathrm{k}(\mathrm{m} / \mathrm{d})$ & $\mathrm{n}(\%)$ & $\mathrm{ne}(\%)$ & $\mathrm{H}(\mathrm{m})$ & $\mathrm{h}_{\mathrm{o}}(\mathrm{m})$ & $\mathrm{i}(\mathrm{m} / \mathrm{m})$ & $v_{d}(\mathrm{~m} / \mathrm{d})$ & $\mathrm{Q}(1 / \mathrm{s})$ \\
\hline 1 & 2092 & 8,1 & 4,0 & 16,70 & 14,1 & 0,033 & 69,03 & 18,997 \\
2 & 2092 & 8,1 & 4,2 & 15,36 & 14,1 & 0,016 & 33,37 & 8,558 \\
3 & 2092 & 8,1 & 4,4 & 15,39 & 14,1 & 0,016 & 34,16 & 8,774 \\
\hline
\end{tabular}

te dispersivo. El menor valor en la velocidad de la fracción móvil del Ensayo 3 en relación al Ensayo 2 es el resultado de una mayor fracción móvil para un caudal muy similar ( $v_{d}$ similares). Los valores de velocidad de la fracción móvil obtenidos con el modelo MIM se encuentran en el rango de valores obtenidos con los modelos Kreft y Zuber (1978) y Lapidus y Amundson (1952), y muestran la misma tendencia de disminución del Ensayo 1 al ensayo 3. En el cuadro 5 se muestran los valores de los parámetros hidráulicos y las condiciones de flujo estimadas para las fechas de ensayo según los resultados del modelo MIM.

En el mismo cuadro se presentan los valores de porosidad total y porosidad efectiva, notándose que la porosidad efectiva está en el rango de los valores encontrados con los modelos de advección-dispersión (cuadro 6). Es decir, debido a que en esos modelos solo se representan los procesos de advección y dispersión, la velocidad empleada es la correspondiente a la fracción que realmente interviene en el trasporte.

La mayor diferencia en los parámetros hidráulicos ocurre para el caso del Ensayo 3, donde el ajuste con el modelo MIM predice un caudal y espesor saturado ligeramente mayores con relación al Ensayo 2. Esta diferencia sin embargo se encuentra dentro del margen de error por la falta de información para esas fechas de ensayo.

\section{VALIDACIÓN DE PARÁMETROS}

En esta sección se compara los valores obtenidos para los parámetros según el modelo MIM con valores reportados en la literatura para ensayos de campo y laboratorio donde se ha aplicado este mismo modelo. En el cuadro 7 se presenta los datos para algunos ensayos reportados.

Es claro del cuadro 7 la gran variabilidad en los valores reportados. Para el caso de materiales fracturados se ha obtenido fracciones móviles entre 0,16 y 0,48 para porosidades totales entre $29 \%$ y $33 \%$ respectivamente. Para porosidades del orden de $13 \%$ se obtuvo fracciones móviles mayores $(0,74-0,84)$. Los valores de 0,49 a 0,54 obtenidos por los autores de esta publicación se encuentran dentro del rango reportado considerando todos los ensayos mencionados. En relación al valor $\alpha$, la variabilidad en los datos es mayor y depende tanto de las condiciones del ensayo como de los materiales empleados. Para materiales fracturados se reportan valores entre $0,34-1,13(1 / d)$. Los obtenidos en este trabajo $(0,74-1,37)$ presentan magnitudes similares.

En relación a los valores de dispersividad, el trabajo de Gelhar, Welty y Rehfeldt (1992) es una referencia clásica. Dichos autores, a través del análisis de valores de dispersión reportados en la

Cuadro 6

Parámetros hidráulicos según modelo advección -dispersión (tomado de Macías et al. [2016]).

\begin{tabular}{cccccccc}
\hline Ensayo & $\mathrm{k}(\mathrm{m} / \mathrm{d})$ & $\mathrm{n}(\%)$ & $\mathrm{H}(\mathrm{m})$ & $\mathrm{h}_{\mathrm{o}}(\mathrm{m})$ & $\mathrm{i}(\mathrm{m} / \mathrm{m})$ & $v_{d}(\mathrm{~m} / \mathrm{d})$ & $\mathrm{Q}(\mathrm{l} / \mathrm{s})$ \\
\hline 1 & $2005-2092$ & $3,6-4,3$ & 16,7 & 14,1 & 0,033 & $66,17-69,03$ & $18,21-18,99$ \\
2 & $2005-2092$ & $3,6-4,3$ & $15,29-15,36$ & 14,1 & $0,015-0,0159$ & $30,18-33,32$ & $7,7-8,5$ \\
3 & $2005-2092$ & $3,6-4,3$ & $15,24-15,31$ & 14,1 & $0,0145-0,0154$ & $28,99-32,13$ & $7,4-8,2$ \\
\hline
\end{tabular}


Cuadro 7

Parámetros de transporte según modelo MIM reportados en la literatura.

\begin{tabular}{|c|c|c|c|c|c|c|c|}
\hline Ensayo & Material & Trazador & ntotal & $\begin{array}{l}\text { Veloc. poros } \\
\qquad(\mathrm{m} / \mathrm{d})\end{array}$ & $\phi_{\mathrm{m}}$ & $\alpha(1 / d)$ & Referencia \\
\hline Lab. & $\begin{array}{l}\text { Lutita alterada } \\
\text { fracturada }\end{array}$ & Bromuro & 0,29 & -- & 0,48 & 1,13 & Kuntz, 2010 \\
\hline Lab. & Suelo limoso & Bromuro & $\begin{array}{c}0,46- \\
0,49\end{array}$ & $\begin{array}{c}3,84 \times 10^{-3}- \\
0,021\end{array}$ & $0,33-0,70$ & $0,22-1,01$ & Johnson, 2004 \\
\hline Lab. & Suelo agregado & Tritio & 0,58 & $0,14 *$ & 0,75 & 0,48 & Nkedi-Kizza et al., 1983 \\
\hline Lab. & Arena fina & Bromuro & 0,41 & 0,018 & 0,80 & 0,01 & Pace, 2003 \\
\hline Lab. & $\begin{array}{l}\text { Lutita alterada } \\
\text { fracturada }\end{array}$ & Bromuro & 0,33 & 3,66 & 0,16 & 0,34 & Mayes et al., 2003 \\
\hline Lab. & Arena arcillosa & Tritio & 0,57 & $1,13-11,12$ & $0,43-0,76$ & $12,75-51,7$ & Seyfried y Rao, 1987 \\
\hline Lab. & Suelo agregado & Bromuro & $\begin{array}{c}0,31- \\
0,36\end{array}$ & -- & $0,11-0,27$ & $2,20-4,65$ & Kohne et al., 2004 \\
\hline Lab. & $\begin{array}{l}\text { Mezcla arcilla } \\
\text { grava y arena }\end{array}$ & $\begin{array}{l}\text { Cloruro de } \\
\text { sodio }\end{array}$ & 0,37 & 14 & 0,61 & 0,10 & Guangyao et al., 2009 \\
\hline Lab. & $\begin{array}{c}\text { Fractura en } \\
\text { flexiglas rellena } \\
\text { con diversos ma- } \\
\text { teriales } 0,5 \mathrm{~mm} \text { a } \\
\quad 0,5 \mathrm{~cm}\end{array}$ & $\begin{array}{l}\text { Cloruro de } \\
\text { sodio }\end{array}$ & 0,40 & 1324-1909 & $0,91-0,96$ & $23-394$ & Qian et al, 2011 a \\
\hline Lab. & $\begin{array}{c}\text { Fractura en } \\
\text { flexiglas rellena } \\
\text { con diversos ma- } \\
\text { teriales }\end{array}$ & $\begin{array}{l}\text { Cloruro de } \\
\text { sodio }\end{array}$ & 0,40 & $532,2-2329,9$ & $0,73-0,99$ & $\begin{array}{c}2125-2,9 \mathrm{x} \\
10^{5}\end{array}$ & Qian et al., 2011 b \\
\hline Lab. & $\begin{array}{l}\text { Arena fina con } \\
\text { macroporos }\end{array}$ & Cloruro & 0,40 & $0,36-0,84$ & $0,39-0,83$ & $0,02-57,6$ & Youngho, 2006 \\
\hline Lab. & Arena & $\begin{array}{l}\text { Cloruro de } \\
\text { sodio }\end{array}$ & 0,45 & 1,84 & 0,99 & 2,28 & Padilla et al., 1999 \\
\hline Campo & $\begin{array}{l}\text { Sedimentos de } \\
\text { tilitas y glacial } \\
\text { fluvial }\end{array}$ & Bromuro & $\begin{array}{c}0,32- \\
0,37\end{array}$ & -- & $0,24-0,69$ & $1,92-6,20$ & Hellerich et al., 2003 \\
\hline Campo & $\begin{array}{c}\text { Limo arcilloso } \\
(\text { loess })\end{array}$ & Tritio & 0,20 & $1,8 \times 10^{-3 *}$ & 0,30 & $\begin{array}{c}1,64 \times 10^{-3}- \\
2,74 \times 10^{-5}\end{array}$ & Gvirtzman et al., 1988 \\
\hline $\begin{array}{l}\text { Campo } \\
\text { Infiltró- } \\
\text { metro }\end{array}$ & $\begin{array}{c}\text { Suelo originado } \\
\text { de depósitos gla- } \\
\text { ciales }\end{array}$ & Fluoruros & 0,40 & 9,33 & 0,60 & 14,0 & Casey et al.,1998 \\
\hline $\begin{array}{c}\text { Campo } \\
\text { Lisímetro }\end{array}$ & $\begin{array}{c}\text { Arena fina no } \\
\text { agregada }\end{array}$ & Bromuro & 0,13 & 7,96 & $0,74-0,84$ & $\begin{array}{c}0,34 \times 10^{-9}- \\
7 \times 10^{-4}\end{array}$ & Ze-Yuan Zou, 1999 \\
\hline
\end{tabular}

*velocidad de fracción móvil $\left(v_{m}\right) ; \phi_{\mathrm{m}}$ fracción móvil, $\alpha$ tasa de trasferencia

literatura para ensayos de campo realizaron una compilación para determinar la relación entre la escala del ensayo y el valor de la dispersividad. Clasificaron la información de campo en relación a la confiabilidad de los datos, obteniendo una clara correlación entre el aumento de la escala del ensayo con el aumento de la dispersividad longitudinal. Según esta correlación, para una escala de 50 a $100 \mathrm{~m}$, los valores de dispersividad con mayor confiablidad están en el rango de 0,35 a $5 \mathrm{~m}$. Los valores obtenidos en esta publicación (para una escala de 78,8 m) tanto con 
los modelos advectivos-dispersivos como el MIM $(0,34-5,63)$ se localizan en este rango.

Para el caso de Costa Rica, algunos valores de dispersividad se han reportado para los materiales volcánicos localizados en la zona del Proyecto Geotérmico Miravallles, obtenidos a partir de ensayos de trazador. Estos valores oscilan entre 15 a $21 \mathrm{~m}$ (para una escala de 800-1400m, Parini, Acuña y Laudiano [1996]) y 54 a 690 m (para una escala de 1000-1300 m, Torres-Mora y Axelsson, [2015]). Sanford at al. (1995) reportan valores de dispersividad longitudinal para lavas del volcán Poas del orden de $50 \mathrm{~m}$ para una escala de 3,5 $\mathrm{km}$, al evaluar la posible conexión del cráter del volcán con zonas de nacientes con aguas ácidas.

Los valores de permeabilidad estimados en este trabajo para el acuífero $(\mathrm{K}=2005-2092 \mathrm{~m} / \mathrm{d}$ equivalentes a $2,32-2,42 \mathrm{~cm} / \mathrm{s}$ ), a pesar de parecer muy altos, se encuentran dentro del rango mostrado por Hoek y Bray (1981) para rocas fracturadas con juntas abiertas $(1-10 \mathrm{~cm} / \mathrm{s})$, y aún menores para la categoría de rocas altamente fracturadas $(10-100 \mathrm{~cm} / \mathrm{s})$. Valores altos de permeabilidad en basaltos fracturados obtenidos a partir de ensayos de trazador en campo han sido también reportados en la literatura. Daly (1982) indica valores de hasta $4,74 \mathrm{~cm} / \mathrm{s}$; Martial, Join y Coudray (2000) reportan permeabilidades en el rango de 0,9 a $3,7 \mathrm{~cm} / \mathrm{s}$ para un valor promedio de $1,2 \mathrm{~cm} / \mathrm{s}$; Levitt et al (2005) reportan valores de $0,98 \mathrm{~cm} / \mathrm{s}$. Anderson, Kuntz y Davis (1999) a partir del análisis de ensayos de bombeo en pozos localizados en el acuífero Snake River Plain en Idaho, Estados Unidos, correlacionan las altas permeabilidades obtenidas en flujos basálticos blocosos tipo pahoehoe $(0,035$ hasta $11,29 \mathrm{~cm} / \mathrm{s}$ ) con su cercanía a las fisuras eruptivas que les dieron origen.

\section{CONCLUSIONES}

Los ajustes obtenidos con el modelo de doble porosidad (MIM) fueron satisfactorios tanto para representar el arribo del trazador como la asimetría en la curva de llegada representada por una cola de decaimiento. Esta asimetría no pudo ser reproducida con el empleo de modelos advectivos-dispersivos de porosidad simple como se muestra en Macías, Vargas y Frutos (2016), indicando que durante el transporte del trazador a lo largo del acuífero estudiado, no solamente actuaron la advección y la dispersión, sino que otros procesos estuvieron involucrados. De acuerdo con la conceptualización del modelo MIM, dichos procesos adicionales responderían al retardo temporal que sufre el soluto debido a desequilibrios físicos causados por fricción y/o turbulencia en las paredes de las fracturas. Estos resultados indican la importancia de emplear modelos de doble porosidad para el análisis e interpretación del transporte de solutos en sistemas de acuíferos fracturados. El empleo de modelos de porosidad simple puede permitir la correcta determinación del tiempo de arribo de un soluto, sin embrago puede fallar para determinar el tiempo de permanencia dentro del acuífero, al no poder representar las colas de decaimiento. Este punto es de gran importancia especialmente para la evaluación de proyectos de remediación en este tipo de materiales, tan comunes en la región centroamericana.

Los parámetros de transporte del modelo MIM obtenidos a partir del empleo de un programa de computador (que permitió ajustar los datos de campo con una curva teórica), sugieren que solamente el $50 \%$ de la porosidad total contribuyó efectivamente al transporte advectivo-dispersivo (fracción móvil), y que el otro 50\% se comportó como un reservorio transitorio para el trazador (fracción inmóvil), siendo liberado o transferido hacia la fracción móvil de manera gradual. La tasa con que se realizó esta transferencia fue diferente entre los tres ensayos en dependencia de las condiciones de flujo imperantes, observándose una mayor tasa para el primer ensayo cuando el caudal de acuífero era mayor, y una menor tasa para el tercer ensayo aun para una velocidad ligeramente superior a la del segundo ensayo. Para el tercer ensayo debería esperarse una mayor tasa de transferencia en comparación con el segundo ensayo. Para explicar esta discrepancia, se propone como hipótesis que la posible mayor concentración inyectada durante el Ensayo 3 contribuyó a disminuir la rapidez en la transferencia de trazador al homogenizar la concentración en las fracturas y crear un menor gradiente de concentración entre las fracciones móvil e inmóvil. Esto contribuyó a 
una mayor fracción móvil en comparación con el segundo ensayo, permitiendo que mayor cantidad de trazador fuera transportada, explicando de esta forma el arribo más temprano detectado durante el Ensayo 3 en comparación con el Ensayo 2. El análisis de acuerdo con el modelo de porosidad simple (Macías, Vargas y Frutos, 2016) sugería que este arribo más temprano era producto de una mayor difusión, sin embargo el empleo del modelo MIM indica que esto se debe a una mayor área de transporte y por consiguiente mayor dispersión mecánica, lo cual parece estar más de acuerdo con los valores similares de velocidad de Darcy estimados para las fechas de estos dos ensayos.

Los valores de los parámetros representativos del modelo MIM obtenidos en este trabajo, así como las propiedades hidráulicas estimadas para el acuífero se encuentran dentro del rango de valores reportados por otros autores. Esto es, a pesar de las limitaciones en la información de campo y en la caracterización inadecuada de los ensayos para una interpretación numérica (no era el objetivo de los mismos) fue posible establecer un marco conceptual y numérico para explicar los tres ensayos.

Los autores de esta publicación consideran que este trabajo aporta al conocimiento en el área centroamericana en dos aspectos esenciales: primero, en la determinación de valores numéricos de parámetros de transporte para el acuífero en análisis, los cuales pueden ser utilizados para estudios posteriores de evaluación de contaminación o remediación o alternativamente extrapolados a otros acuíferos similares bajo un buen criterio profesional, y segundo en el planteamiento de una metodología de utilidad para los consultores en hidrogeología o ambiental para el análisis más adecuado de acuíferos volcánicos fracturados o de doble porosidad.

\section{REFERENCIAS BIBLIOGRÁFICAS}

Anderson, S. R., Kuntz, M. A., y Davis, L. (1999). Geologic controls of hydraulic conductivity in the Snake River Plain Aquifer at and near the Idaho National Engineering and Environmental Laboratory, Idaho. Idaho: Geological Survey, Water-Resources Investigations Report 99-403.
Bouyère, S., Carabin, G. y Dassargues, A. (2005). Influence of Injection Conditions on Field Tracer Experiments. Ground Water, 43(3), 389-400.

Casey F., Logsdon S., Horton R., y Jaynes Dan B. (1998). Measurement of Field Soil Hydraulic and Solute Transport Parameters. Soil Science Society of America Journal, 62, 1172-1178.

Daly, C. (1982). Evaluation of procedures for determining selected aquifer parameters. CRREL Report 82-41. Hanover, N.H.: U.S. Army Corp of Engineers. U.S. Army Toxic and Hazardous Materials Agency.

Fetter., C. W. (1993). Contaminant hydrogeology. Nueva Jersey: Prentice Hall.

Gelhar L., Welty C. y Rehfeldt K. (1992). A critical review of data on field-scale dispersion in aquifers. Water Resources Research, 28(7), 1955-1974.

Griffioen, J. W., Barry, D. A. y Parlange, J. Y. (1998). Interpretation of two-region model parameters. Water Resources Research, 34(3), 373-384.

Guangyao G., Shaoyuan F., Hongbin Z., Guanhua H., y Xiaomin, M. (2009). Evaluation of Anomalous Solute Transport in a Large Heterogeneous Soil Column with MobileImmobile Model. Journal of Hydrologic Engineering, 9, 966-974.

Gvirtzman H., Paldor N., Magaritz M. y bachmat Y. (1988). Mass Exchange Between Mobile Freshwater and Immobile Saline Water in the Unsaturated Zone. Water Resources Research, 24(10), 1638-1644.

Hellerich, L. A., Oates, P. M, Johnson, C. R., Nikolaidis, N. P., Harvey C. F. y Gschwend, P. M. (2003). Bromide transport before, during, and after colloid mobilization in push-pull tests and the implications for changes in aquifer properties. Water Resources Research, 39(10), 15-1: 15-11. 
Hoek, E. y Bray, J. W. (1981). Rock Slope Engineering ( ${ }^{\text {ra }}$ ed.). Londres: Institution of Mining and Metallurgy.

Johnson, A. (2004). Saturated transport of atrazine under two tillage systems. En D. E. Stott, R. H. Mohtar y G. C. Steinhardt (eds), Sustaining the global farm. 10th International Soil Conservation Organization Meeting (pp. 283-287). West Lafayette, IN: International Soil Conservation Organization.

Kohne, J., Kohne, S., Mohanty, B. y Simunek, J. (2004). Inverse Mobile-Immobile Modeling of Transport During Transient Flow: Effects of Between-Domain Transfer and Initial Water Content. Vadose Zone Journal, 3, 1309-1321.

Kreft, A. y Zuber, A. (1978). On the physical meaning of the dispersion equation and its solutions for different initial and boundary conditions. Chemical Engineering Science, 33, 1471-1480.

Kuntz, B. (2010). Laboratory, field, and modeling analysis of solute transport behavior at the shale hills critical zone observatory (Tesis de maestría inédita). Pennsylvania State University, Pennsylvania, Estados Unidos.

Lapidus, L. y Amundson, N. R. (1952). Mathematics of adsorption in beds. VI. The effects of longitudinal diffusion in ion exchange and chromatographic columns. Journal of Physical Chemistry, 56, 984-988.

Levitt, D. G., Newell, D. L., Stone, W. F. y Wykoff, D. S. (2005). Surface WaterGroundwater Connection at the Los Alamos Canyon Weir Site: Part 1. Monitoring Site Installation and Tracer Tests. Vadose Zone Journal, 4, 708-717.
Macías, J.,Vargas, A. y Frutos, F. (2016). Estimación de parámetros hidrodinámicos en un acuífero volcánico libre fracturado en Costa Rica. Parte I. Revista Geológica de América Central, 55, 167-183.

Martial J. S., Join J. L. y Coudray, J. (2000). Hydrodynamical investigations in a recent volcanic aquifer (Reunion Island) using single well tracer test. Groundwater-Past achievements and Future challenges (pp. 221-224). Presentado en Proc. XXX IAH Congress on Groundwater, Cape Town, South Africa.

Mayes, M. A., Jardine, P. M., Mehlhorn, T. L., Bjornstad, B. N., Ladd, J. L. y Zachara, J. M. (2003). Transport of multiple tracers in variably saturated humid region structured soils and semi-arid region laminated sediments. Journal of Hydrology, 275(3-4), 141-161.

Nkedi-Kizza P., Biggar J. W., van Genuchten M. T., Wierenga P. J., Selim, H. M., Davidson J. M. y Nielsen D. R. (1983). Modeling Tritium and Chloride 36 transport through and aggregated oxisol. Water Resources Research, 19(3), 691-700.

Pace M. N., Mayes M. A., Jardine P. M., Mehlhorn T. L., Zachara J. M. y Bjornstad, B. N. (2003). Quantifying the Effects of SmallScale Heterogeneities on Flow and Transport in Undisturbed Cores from the Hanford Formation. Vadose Zone Journal, 2, 664-676.

Padilla Y. I., Jim Yeh, T. C., y Conklin, M. H. (1999). The effect of water content on solute transport in unsaturated porous media. Water Resources Research, 35(11), 3303-3313.

Parini M., Acuña J. y Laudiano M. (1996). Reinjected water return at Miravalles geothermal reservoir, Costa Rica: 
Numerical modelling and observations (pp. 127- 134). Presentado en Proc. Twentyfirst Workshop on Geothermal Reservoir Engineering. Stanford University, Stanford, California.

Qian J. Z. Chen Z., Zhan H. B. y Luo. S. H. (2011a). Solute transport in a filled single fracture under non-Darcian flow. International Journal of Rock Mechanics y Mining Sciences, 48(2011), 132-140.

Qian J. Zhan H., Chen Z., y Ye, H. (2011b). Experimental study of solute transport under non-Darcian flow in a single fracture. Journal of Hydrology, 399, 246-254.

Raven, K., Novakowski, K. y Lapcevic, P. (1988). Interpretation of field tracer test of a single fracture using a transient solute storage model. Water Resources Research, 24(12), 2019-2032.

Sanford W., Konikow L., Rowe G., y Brantley, S. (1995). Groundwater transport of craterlake brine at Poas Volcano, Costa Rica. Journal of Volcanology and Geothermal Research, 64, 269-293.
Seyfried, M. S. y Rao, P. (1987). Solute transport in undisturbed columns of an aggregated tropical soil: Preferential flow effects. Soil Science Society of America Journal, 51(6), 1434-1444.

Torres-Mora Y. y Axelsson, G. (2015). Chemical tracer test in Las Pailas Geothermal Field, Costa Rica. Presentado en Proc. World Geothermal Congress 2015, Melbourne, Australia. Recuperado de Disponible en: http://www.geothermal-energy.org/pdf/ IGAstandard/WGC/2015/14070.pdf

van Genuchten, M. (1981). Non-Equilibrium transport parameters from miscible displacement experiments. Riverside, California: U.S. Salinity Laboratory. Department of Agriculture Science and Education Administration. Research Report 119.

Youngho, S. (2006). Characterizing the fate and transport of solutes in soil (Tesis de doctorado inédita). The University of Tennessee, Knoxville.

Ze-Yuan Z. (1999). Determination of flow and transport properties in a deep unsaturated soil profile (Tesis de doctorado inédita). University of Arizona, Arizona, Estados Unidos. 
